\title{
Procesos constructivos de contenidos de paz. Una mirada a emisoras del municipio de Bello (Colombia)*
}

\author{
Alma Leonora García Gómez \\ José Julián Herrera Pulgarín ${ }^{* * \star}$ \\ Sergio Alonso Arenas Ceballos***
}

Recibido: 2020-02-18 • Enviado a pares: 2020-03-09

Aprobado por pares: 2020-05-05 - Aceptado: 2020-05-22

https://doi.org/10.22395/angr.v19n37a10

\begin{abstract}
Resumen
El propósito de este trabajo es caracterizar los procesos de construcción de contenidos de paz en los medios radiales San Buenaventura Estéreo y Radio B del municipio de Bello (Antioquia, Colombia), conforme con las directrices sugeridas por parte de organismos nacionales e internacionales en pro de la creación de relatos que coadyuven a la memoria histórica de esta localidad. Se justifica su realización en tanto parte de la tipología de investigación constructiva sobre la paz que permite comprender los modos en que los productores radiales y los oyentes de este lugar, reconocido como escenario de violencia, edifican la cultura de paz a partir de los relatos reconciliadores de sus propias violencias. La metodología sigue el constructivismo de paz, por lo que el estudio es cualitativo y descriptivo con un muestreo no probabilístico por conveniencia; al ser pocas las emisoras, solo las elegidas contaban con producción en esta materia. Se emplearon técnicas de producción de datos como revisión documental de la categoría contenidos de paz en las realizaciones radiales: Por las calles de mi barrio, con líderes barriales; Locutores por la paz, con niños; y En clave de paz, con actores del conflicto. Además, se hicieron entrevistas semiestructuradas y talleres participativos, aplicados a directores y productores de los medios abordados, desde las categorías principios de producción radial y memoria histórica. Como hallazgos se destacan la proactividad de los productores y su interés en la generación de los programas en un contexto donde las capacidades endógenas de los bellanitas operan como recurso de resiliencia. El análisis indica que los nuevos relatos producidos en estos medios contribuyen a la construcción de la memoria histórica del municipio, donde los participantes ven en el medio radial, y su labor, oportunidades de aprendizajes para la vida que pueden fortalecerse con la academia para garantizar su sostenibilidad con la formación a las generaciones más jóvenes.
\end{abstract}

Palabras clave: cultura de paz; programas de radiodifusión; contenido de programas radiales; medios comunitarios; habilidades.

* El artículo hace parte del proyecto de investigación Contenidos y narrativas de paz en los medios comunitarios y locales de los municipios de la zona norte del Valle de Aburrá (Bello, Copacabana, Girardota y Barbosa); desarrollado durante el año 2019 en esta misma localidad como parte de la IV Convocatoria de Proyectos de Ciencia, Tecnología, Creación e Innovación de Menor Cuantía financiada por la Corporación Universitaria Minuto de Dios Seccional Antioquia Chocó, Colombia (Código Cidub CB18-18). La investigación está adscrita al Grupo de Investigación Communicare a través del semillero del mismo nombre, el cual responde a la sublínea investigativa de comunicación, desarrollo, participación y cambio social.

** Magíster en Comunicación, Universidad Pontificia Bolivariana, Medellín, Colombia. Docente e investigador, Corporación Universitaria Minuto de Dios Seccional Antioquia Chocó, Colombia. Líder del Semillero Communicare. Correo electrónico: alma.garcia@uniminuto.edu.co. Orcid: https://orcid.org/0000-0002-1585-1217

*** Candidato a doctorado en Ciencias de la Educación, Universidad Nacional de Rosario, Rosario, Argentina. Docente e investigador, Corporación Universitaria Minuto de Dios Seccional Antioquia Chocó, Colombia. Líder del Semillero Communicare. Correo electrónico: jherrerapul@uniminuto.edu.co. Orcid: https://orcid.org/0000-0001-6815-2930

**** Comunicador Social Periodista, Corporación Universitaria Minuto de Dios, Colombia . Miembro del Semillero Commnicare. Correo electrónico: sarenascebas@uniminuto.edu.co. Orcid: https://orcid.org/0000-00025730-6188 


\title{
Constructive Processes for Peace Contents. A Look to Radio Stations of Bello (Colombia)
}

\begin{abstract}
The purpose of this work is to characterize the peace contents construction processes in the San Buenaventura Estéreo and Radio B radio station of the Bello municipality (Antioquia, Colombia), in accordance with the directions suggested by the national and international organizations in pro for creation of narratives that aid the historical memory of this locality. The study is justified given that it is based upon the constructive research typology that allows the comprehension of how radio producers and audiences in this place, recognized as a violent scenario, are building a peace culture from reconciliatory narratives based on its own violence. The methodology closely follows peace constructivism, the reason why the study is qualitative and descriptive with a convenient non-probabilistic sampling; because of how few the stations are, only those chosen had productions in this line.

Various techniques for data production were employed as a documentary review of the contents for peace category in the radio productions: Por las calles de mi barrio (Through the streets of my neighborhood), with neighborhood leaders; Locutores por la paz (Broadcasters for peace), with children; and En clave de paz (In peace key), with armed conflict actors. Besides, semi-structured interviews were performed in group workshops with directors and producers of the studied stations based on the principles for radio production and historical memory categories. The proactivity of the producers and their interest in generating these programs in a context were the endogenous capacities of the inhabitant of Bello operate as a resilience resource stand out as the main findings. The analysis points towards that the new narratives produced in these media aid the historical memory for peace construction in the municipality, where the participants see in the radio and their duty opportunities for learnings relevant for their lives that can be strengthened with academia for ensuring their sustainability with the education of younger generations.
\end{abstract}

Keywords: peace culture; radio broadcast programs; radio program contents; community media; abilities.

\section{Processos construtivos de conteúdos de paz. Um olhar nas emissoras do município de Bello (Colômbia)}

\begin{abstract}
Resumo
Este trabalho tem o objetivo de caracterizar os processos de construção de conteúdos de paz nas emissoras de rádio San Buenaventura Estéreo e Radio B do município de Bello (Antioquia, Colômbia), conforme as diretrizes das organizações nacionais e internacionais em prol da criação de relatos que contribuem para a memória histórica dessa região. Sua realização é justificada por fazer parte da tipologia de pesquisa construtiva sobre a paz que permite compreender os modos nos quais os produtores de rádio e os ouvintes do lugar, reconhecido como cenário de violência, edificam a cultura da paz a partir dos relatos reconciliadores de suas vivências. A metodologia segue o construtivismo de paz, por isso o estudo é qualitativo e descritivo com uma amostra não probabilística por conveniência; ao serem poucas as emissoras, somente as selecionadas contavam com produção nessa matéria. Foram utilizadas técnicas de produção de dados como revisão documental da categoria "conteúdos de paz" nas realizações radiais: Por las calles de mi barrio, com líderes de bairros; Locutores por la paz, com crianças, e En clave de paz, com atores do conflito. Além disso, foram realizadas entrevistas semiestruturadas e oficinas participativas com diretores e produtores dos meios abordados, a partir das categorias "princípios de produção radial" e "memória histórica". Como achados, são destacados a proatividade dos produtores e seu interesse na geração de programas em um contexto em que as capacidades endógenas dos habitantes de Bello operam como recurso de resiliência. A análise indica que os novos relatos produzidos nesses meios contribuem para a construção da memória histórica do município, em que os participantes veem no meio radial e em seu trabalho oportunidades de aprendizagem para a vida que podem ser fortalecidas com a academia para garantir sua permanência com a formação das gerações mais jovens.
\end{abstract}

Palavras-chave: cultura de paz; programas de radiodifusão; conteúdo de programas radiais; meios comunitários; habilidades. 


\section{Introducción}

El establecimiento de los acuerdos entre la guerrilla de las FARC y el Gobierno de Colombia introduce al país en el contexto de la cultura de la paz, como escenario axiológico y actitudinal, donde se rechaza la violencia y se enfatiza en la prevención del conflicto a partir de acciones dialógicas y de negociación entre sus partes (Unesco, 1999). Una de estas acciones radica en el abordaje que realizan las comunidades mediante la construcción de contenidos que se puedan difundir a través de los medios de comunicación —entre ellos la radio- sin distinción de su tipología, ya que está llamada a consolidar dicha cultura y a enfatizar en su sensibilidad hacia el sentir social y su contribución en la reconstrucción del tejido social (López y Arriola, 2015). Los medios radiales cumplen con el cometido del acuerdo de paz en el numeral 2.2.3 a través del fomento y divulgación de una cultura pacífica con la interacción de todos sus implicados; además de la equidad y el encuentro con los otros después de las brechas causadas por el conflicto (Alto Comisionado para la Paz, 2016).

En este sentido, es conveniente preguntar: ¿De qué modo la comunidad del municipio de Bello (Antioquia), víctima del conflicto armado, se apropia de las disposiciones de organismos nacionales e internacionales con respecto a la construcción de contenidos radiales de paz en pro de la generación de nuevas narrativas? Para resolver esta pregunta, se propone como objetivo general caracterizar los procesos de construcción de contenidos de paz en los medios radiales San Buenaventura Estéreo y Radio B del municipio de Bello (Antioquia, Colombia), según las directrices sugeridas por parte de organismos nacionales e internacionales en pro de la creación de relatos que coadyuven a la memoria histórica de esta localidad. Como objetivos específicos, se pretende identificar los medios y sus rutinas para la producción de contenidos de paz con los públicos participantes; analizar los hallazgos en la óptica de las disposiciones de los organismos que los sustentan y favorecen; además de proponer alternativas que buscan garantizar su sostenibilidad como elaboraciones que contribuyen a la memoria histórica de este municipio.

El contexto elegido obedece a diversas condiciones socio políticas. Si bien no es una zona de gran impacto por el conflicto armado con las FARC, es escenario de diversos periodos del conflicto urbano (Secretaría de Educación y Cultura, 2015). Entre ellos se encuentran: i) la convivencia entre migrantes arribados tras la creación de Fabricato como industria textil, con un campesinado convertido en obreros; ii) la cooptación que hace la cultura del narcotráfico de este tipo de población desarraigada, básicamente la juvenil, lo que conduce a la creación de bandas delincuenciales en los años más críticos de la violencia en el municipio; y iii) el hecho de ser un lugar receptor de una amplia población de personas en situación de desplazamiento vía disputa armada, lo que genera distintos campos de violencia política, de economía de drogas y tensiones sociales (Pecaut, 1997). Junto a ello, se entreteje una trayectoria propia de formas de 
resiliencia con amplios sectores de participación social mediante liderazgos, animación sociocultural, teatro, recreación y corporaciones culturales (Secretaría de Educación y Cultura, 2015). En este contexto, surgen las dos emisoras seleccionadas. Estas son las únicas que cuentan con una parrilla de programación sobre paz en esta localidad: Por las calles de mi barrio, experiencia de la emisora Comunitaria San Buenaventura Estéreo, adscrita a la Parroquia Santa Clara de Asís del municipio de Bello; y las series Locutores por la paz y En clave de paz de la emisora cultural y digital Radio B, relacionada con la Secretaría de Cultura de Bello y su Escuela de Música Juvenil.

La estructura del artículo parte de una aproximación conceptual a la cultura de la paz y el paradigma constructivo de sus contenidos en medios radiales a partir de las disposiciones de la ONU y el Ministerio de Cultura de Colombia; estos organismos son rectores en este tipo de realizaciones comunicativas para el posacuerdo, y orientadores para medios locales y comunitarios. Se incluye el estado del arte más destacado en construcción de contenidos radiales de paz convenientes al estudio, además de las experiencias destacables a las que es necesario acudir a causa del poco tiempo que ajusta el fin de conflicto armado con la guerrilla de las FARC. En segundo lugar, aparecen las precisiones metodológicas donde se establece el tipo de estudio cualitativo, de enfoque descriptivo y desde la línea investigativa de construcción de la paz. En dicho apartado se delimitan el objeto de estudio, el muestreo, la muestra, los instrumentos de recolección de información y las técnicas de análisis de datos convenientes al trabajo. Todo esto se hace con el fin de responder a las tres categorías del estudio: contenidos radiales de paz, principios de producción radial y memoria histórica. El apartado de los resultados permite identificar la producción de contenidos de paz en los medios elegidos, mientras que el análisis revisa los datos encontrados desde las indicaciones de las instituciones nacional y foránea. De esta manera, se culmina con posibles estrategias a adoptar en favor de la memoria histórica bellanita a partir de las producciones radiales de paz.

Desde el ámbito teórico, el tema de la paz y sus contenidos puede mirarse desde tres líneas de investigación: la crítica, la empírica y la constructiva (Galtung, 2008; 2015). Las dos primeras parten de estudiar lo que podría desarrollarse sobre el valor de la paz a partir situaciones existentes; la tercera, en la que se inscribe el presente trabajo, relaciona teorías factibles con valores desde el deber ser, donde se concibe la edificación de la paz a partir del encuentro interdisciplinar en pro de la revisión y la creación conjunta. Desde este paradigma, los contenidos producidos por los medios de comunicación son a la vez marcos conceptuales que se explican en los escenarios en que tienen lugar (Ministerio de Cultura, 2016). En este sentido, propician un horizonte de comprensión alternativo a la violencia cultural (Galtung, 2014), es decir, a la necesidad de cambiar el modo en que se habla del conflicto y sus matices, el lenguaje y significantes empleados en la producción de discursos que hablan del conflicto 
mediante una paz denominada "positiva" o creativa (Galtung, 2014). Asimismo, estos contenidos se pueden expresar desde diversos medios (Escuela de Cultura de Paz, 2011) que se orientan hacia una paz que es imperfecta, pero que sirve para resaltar las acciones que la generan y las interacciones entre estas (Muñoz y Molina, 2010). Este tipo de creaciones requieren valores específicos (Cerdas-Agüero, 2015) a través de procesos formativos en los que estos se ejercitan para gestionar el conflicto (Alí, 2012), sobre todo con la comunicación alternativa (Caridad et al., 2017). Pero no dejan de visibilizarlo a través de noticias positivas y contextualizadas (Espinoza, 2017), sin profundizar en polaridades, con una postura ética diversa (Calderón, 2015). Todo ello sucede en un escenario donde el periodismo de paz, si bien surge como opción de cambio social y responsable con respecto al conflicto (Gouse et al., 2019), todavía es normativo (Espinar y Hernández, 2012), y no ha tenido un despliegue amplio y variopinto en los medios (Calderón, 2015). Los contenidos de paz coadyuvan al ejercicio de la memoria sobre experiencias de violencia y la comunicación de estas, respetando su carácter innegociable, de modo que generan identidad y salud mental social gracias a que posibilitan la reconciliación (Gaborit, 2006). En esta medida, se inscriben como nuevas gramáticas de significación, ya que buscan la justicia léxica ante las experiencias que la violencia les arrebata el sentido y la voz (Acosta, 2019). En esta misma línea, el Ministerio de Cultura de la República de Colombia (2016), en consonancia con las indicaciones de la Unesco (1999), señala cinco principios básicos para tener en cuenta en la producción de contenidos culturales para la paz. Estos principios son el resultado del uso creativo de narrativas diversas e incluyentes con los que se propende hacia nuevos relatos del país:

- La diversidad cultural como variedad de formas en que se transmite el patrimonio artístico y las creaciones de los grupos humanos.

- La inclusión con la acogida favorable de la diferencia más allá de la estima respetuosa del otro.

- La participación relacionada con el desarrollo, el fomento de la ciudadanía y la inclusión cultural.

- El respeto como opción por la multiculturalidad y la interculturalidad.

- El diálogo cultural como agente de la creación y la memoria.

Por su parte, las investigaciones sobre construcción de contenidos radiales de paz, en la óptica de los acuerdos con las FARC, son incipientes. En ellos se privilegia el papel de las radios comunitarias como medios para resignificar los territorios, construir la memoria histórica de sus habitantes y promover la resolución de problemas en el nuevo escenario del posconflicto. En este sentido, los oyentes no solo son concebidos como sujetos de información, sino como participantes de la misma (Zúñiga y Grattan 2017). Si bien se apuesta a la edificación de la paz desde la reconciliación, con 
un valor marcado en las Juntas de Programación y los Comités Temáticos Locales, se encuentran serias dificultades en los órdenes administrativo y de continuidad en las políticas de gobierno (Vega, Tapias y Pérez, 2019). Se encuentran así los casos de las comunidades indígenas en el Cauca (Useda y Carillo, 2018) y las experiencias radiales de Soacha (Reyes, 2017), Funza y Bogotá, Cundinamarca (Espitia, 2018); Tenjo, Palmira (Belalcázar, Botero y Ospina, 2019); Simití, Bolívar (Moreno, 2019); Briceño y San Pedro de los Milagros, Antioquia (Vega, Tapias y Pérez 2019). En relación con otros acuerdos de paz ya establecidos, se destacan tres experiencias modelo en donde la cocreación y el diseño participativo de programas fueron las constantes: Radio Andaquí, Caquetá (González y Rodríguez, 2006); Radio nacional de Colombia en los Montes de María, en Bolívar y Sucre (Vega y Bayuelo, 2008); y la Red de Radios Comunitarias del Magdalena Medio, Aredmag (Cadavid y Moreno, 2009; Vega y Rodríguez, 2009).

En lo referente a experiencias radiales con contenidos de paz destacan Sintonízate con la paz de la fundación Arco Iris. En el caso de los niños, resaltan las siguientes producciones: Tripulantes de la Universidad Distrital Francisco José de Caldas, Bogotá; El lazo de la diversión de la emisora Radio Semillas de Tibasosa, Boyacá; Voz infantil, hola juventud de la Voz de la Costa, Barranquilla; Infancias con voz de la Secretaría Distrital de Integración y el Instituto Distrital de la participación y Acción Comunal (Idpac), Bogotá (Señal Colombia, 2016).

\section{Metodología}

Como ejercicio de la línea investigativa de construcción de la paz, el estudio propuesto fue de tipo cualitativo y de enfoque descriptivo, de modo que se produjeron registros pormenorizados a partir de los generadores de contenidos (Taylor y Bogdan, 1986). La población de emisoras radiales en el municipio de Bello no era amplia. Tan solo tenía tres, de las cuales solo podíamos elegir las que tenían producción de contenidos de paz. Dadas las condiciones territoriales y sociales, el muestreo no probabilístico fue por conveniencia con dos criterios de entrada: contar con una parrilla de programación con creación propia y estar en proceso de ejecución o finalización de algún tipo de producción en materia de paz o temáticas afines. Con base en ello, la etapa preliminar del estudio recurrió a una lista de chequeo para garantizar los elementos de entrada. Así, fueron seleccionados dos medios radiales y sus respectivos programas: San Buenaventura Estéreo, emisora comunitaria creada en el año 1995 (95.4 FM), con el programa Por las calles de mi barrio; y Rabio B, emisora cultural digital surgida en el año 2017 (sitio web radiob.co) con las series Locutores por la paz y En clave de paz. Conforme con el tipo de investigación, los instrumentos de recolección de información incluyeron: exploración documental sobre su conformación y realizaciones en materia de paz a fin de identificar los medios y las rutinas de producción; entrevistas semiestructuradas a los directores (dos unidades de análisis) y productores de los programas radiales con contenidos de paz (tres unidades). A partir de estas se eligió el número de producciones 
a revisar: dos del programa de la emisora comunitaria y dos por cada una de las series de la emisora digital. La categoría directriz contenidos de paz fue desplegada en tres subcategorías: las narrativas o discursos empleados por los realizadores de los programas; las rutinas de producción o prácticas de construcción noticiosas (newsmaking) en tanto se efectúan acciones de periodismo de paz; y las formas de participación logradas con las creaciones radiales. El análisis de los contenidos de paz, bajo las indicaciones de organismos competentes, recurrió a una matriz de análisis de contenidos con el fin de subrayar sus logros y prospectivas desde la categoría principios de producción radial. Por su parte, la generación de estrategias para la contribución a la memoria histórica del municipio, desde los contenidos encontrados, empleó un taller de diseño participativo con los directores y productores de las emisoras. Esto se hizo con el ánimo de generar propuestas que pudieran potenciar el trabajo adelantado por los medios de la mano de la academia. Para corresponder con el sentido constructivo de la paz y la categoría memoria histórica, el taller fue formulado con temas específicos sugeridos por los actores implicados, donde discutieron estrategias a adoptar para continuar con los esfuerzos de construir la paz desde las localidades y desde las voces de los ciudadanos. Con los resultados obtenidos, se elaboró un documento para ser socializado y aprobado con sus beneficiarios. De este modo, se estableció una agenda de acciones concretas de la mano de los investigadores que acompañaron el proceso para fortalecer los logros y generar alternativas frente a los contenidos de paz producidos. Los datos fueron recogidos entre los meses de febrero y abril del año 2019, y procesados a través de Excel, con el propósito de organizar tablas con aspectos propios de la investigación. Además, se evaluó su utilidad analítica (Kalman y Rendón, 2016). Se elaboraron descriptores gráficos para una mayor comprensión de los resultados, y el análisis recurrió a la metodología hermenéutico-dialéctica y constructiva de la paz.

\section{Resultados}

En la emisora San Buenaventura Estéreo (95.4 FM), se encontró que el interés sobre el trabajo por la paz, asociado a su filiación institucional de tipo confesional católica mas no ideológica, motivó el paso de programas de evangelización y reflexión sobre la moral hacia la experiencia de creación conjunta con la comunidad; en este caso, con asuntos directamente de paz, en la óptica del posacuerdo. El medio contaba con un espacio denominado "Lectio Divina", donde se hablaba sobre asuntos de paz y convivencia conforme a la enseñanza de la biblia. No obstante, el contacto con entidades públicas y otras organizaciones — quienes ofrecieron información sobre la cultura de paz y la necesidad de mantener el sentido comunitario propio de la emisora tras quedar bajo la supervisión de la parroquia y colegios anexos- generaron la opción de integrar las Juntas de Acción Comunal (JAC) de los barrios en las dinámicas de producción de contenidos de paz. En este contexto surgió Por las calles de mi barrio, en el cual se da la palabra a los líderes de dichas juntas para incluir sus relatos y los 
relatos de sus vecinos, con los cuales se reflejan las distintas posturas frente al conflicto. Con tres sesiones pregrabadas en las instalaciones de la emisora, a comienzos del año 2019, pasó a enfocarse en un trabajo de campo por las calles del municipio, que se entremezcla con la producción de la emisora. El asiduo compromiso de los líderes, sumado a su interés de incluir en la agenda pública las problemáticas de sus barrios, fueron las constantes destacadas.

La revisión de la subcategorías (tabla 1) en los contenidos del programa elegido, permitió establecer que este programa estaba dirigido al público en general, pues tenía narrativas que mostraron mensajes formadores de la no violencia a partir de acciones propias de los vecinos. En este sentido, se abordaron temáticas específicas para suplir la ausencia del Estado y buscar la no repetición. En estas rutinas de producción se dieron formas de cocreación entre los líderes de las Juntas de Acción Comunal y los directores radiales. Por otra parte, la participación de los oyentes era asidua y empleaban diferentes canales.

Tabla 1. Contenidos de paz en el programa radial Por las calles de mi barrio

\begin{tabular}{|c|c|c|c|c|}
\hline \multirow[b]{2}{*}{ Programa/serie } & \multirow[b]{2}{*}{ Actores } & \multicolumn{3}{|c|}{ Contenidos de paz } \\
\hline & & Narrativas & $\begin{array}{l}\text { Rutinas de } \\
\text { producción }\end{array}$ & Participación \\
\hline 09/03/2019 & $\begin{array}{l}\text { Presidentes } \\
\text { JAC de los ba- } \\
\text { rrios Mirador y } \\
\text { Alpes. }\end{array}$ & $\begin{array}{l}\text { Ausencia del Estado y for- } \\
\text { mas de congregación co- } \\
\text { munitaria. }\end{array}$ & \multirow{3}{*}{$\begin{array}{l}\text { Programas pre- } \\
\text { grabados con } \\
\text { preproducción } \\
\text { en los barrios. } \\
\text { Uso de vox pop } \\
\text { sobre temas im- } \\
\text { portantes, testi- } \\
\text { monios de acto- } \\
\text { res de los barrios. } \\
\text { Interacción con el } \\
\text { productor, quien } \\
\text { planifica con los } \\
\text { invitados. }\end{array}$} & \multirow{3}{*}{$\begin{array}{l}\text { Indirecta por parte } \\
\text { de los oyentes de } \\
\text { las comunidades } \\
\text { invitadas con la } \\
\text { escucha del pro- } \\
\text { grama. } \\
\text { Directa con opi- } \\
\text { niones de la comu- } \\
\text { nidad a través de } \\
\text { las redes sociales } \\
\text { de la emisora o la } \\
\text { intervención en los } \\
\text { pregrabados en te- } \\
\text { rritorio. }\end{array}$} \\
\hline \multirow[t]{2}{*}{ 08/06/2019 } & \multirow{2}{*}{$\begin{array}{l}\text { Líder JAC sec- } \\
\text { tor Maxicolbe, } \\
\text { coordina- } \\
\text { dor de Mesa } \\
\text { de Trabajo } \\
\text { Comuna } 7 .\end{array}$} & $\begin{array}{l}\text { Descripción de violencias } \\
\text { y capacidades endógenas } \\
\text { de los vecinos para con- } \\
\text { trarrestarlas. Prácticas de } \\
\text { arraigo y sinergia de la JAC } \\
\text { con la comunidad. }\end{array}$ & & \\
\hline & & $\begin{array}{l}\text { Descripción de problemá- } \\
\text { ticas de seguridad, salud y } \\
\text { medio ambiente. }\end{array}$ & & \\
\hline
\end{tabular}

Fuente: elaboración propia.

Por su parte, la parrilla de programación de Radio B surgió por vocación de cada uno de los actores involucrados en la realización, esto es, se hizo de acuerdo con sus gustos, con la apertura y tipo de razón social de la emisora cultural. Con el interés de difundir los logros y acciones de la Escuela de Música del municipio de Bello, Radio B abrió los micrófonos a diferentes líderes, sectores y colectivos — no solo de la localidad, sino de otras localidades cercanas-, donde cada uno asumió la producción de series radiales. Entre estos líderes se contaron profesionales que, aunque no tenían 
conocimientos certificados en radio, quisieron difundir, con sus trayectorias en el trabajo por la paz, los mensajes de diferentes actores y colectivos sociales respecto al tema, a partir de sus saberes específicos y experiencias de vida. Aunque cada uno decidió presentar un formato diferente de serie, con participantes y públicos objetivos también particulares, coincidieron en una concepción de paz a partir de las versiones de las personas y su postura frente al conflicto, más allá de una postura academicista. En el caso de la serie Locutores por la paz, la vida y la convivencia en familia fueron los referentes para los productores menores de edad. Por otro lado, en la serie En clave de paz hicieron lo propio a partir del contexto y voces de los actores de la guerra. Estas realizaciones no fueron fáciles de hacer en sus inicios. Según los productores, la razón de ello se debe al perfil de los participantes vinculados. En este sentido, se requirió preparación y acuerdos para acceder a las dinámicas de la radio, de manera especial con los menores, al igual que una postura de respeto en el momento de referir asuntos álgidos del conflicto.

La revisión de las subcategorías en Locutores por la paz (tabla 2) permitió encontrar relatos construidos desde voces infantiles, en compañía de los adultos, acerca de la generación de entornos favorables para la paz. Las rutinas de producción incluyeron la garantía de los derechos de los menores y la asistencia de los padres a las grabaciones en cabina. El diseño de libretos por parte del director del programa fue enriquecido con comentarios de los niños antes y durante la grabación, por lo que se pudo contar como una forma de participación.

Tabla 2. Contenidos de paz en la serie radial Locutores por la paz

\begin{tabular}{|c|c|c|c|c|}
\hline \multirow{2}{*}{ Programa/serie } & \multirow{2}{*}{ Actores } & \multicolumn{3}{|c|}{ Contenidos de paz } \\
\hline & & Narrativas & Rutinas de producción & Participación \\
\hline $\begin{array}{l}\text { Capítulo } 1 . \\
\text { El amor de } \\
\text { padres }\end{array}$ & \multirow{3}{*}{$\begin{array}{l}\text { Productor, tres } \\
\text { niñas copro- } \\
\text { ductoras y pa- } \\
\text { dres de familia. }\end{array}$} & $\begin{array}{l}\text { Necesidad de las buenas } \\
\text { relaciones con los padres } \\
\text { para la convivencia. }\end{array}$ & \multirow{3}{*}{$\begin{array}{l}\text { Programas pre- } \\
\text { grabados con } \\
\text { preproducción de } \\
\text { algunos vox popy } \\
\text { preguntas de ter- } \\
\text { ceros, interven- } \\
\text { ción constante de } \\
\text { las menores, apo- } \\
\text { yo en la postu- } \\
\text { ra de los padres. } \\
\text { Interacción con el } \\
\text { productor, quien } \\
\text { establece un li- } \\
\text { breto y lo pone a } \\
\text { consideración de } \\
\text { los participantes. }\end{array}$} & \multirow{3}{*}{$\begin{array}{l}\text { Indirecta por parte } \\
\text { de los oyentes de } \\
\text { las comunidades } \\
\text { invitadas a la escu- } \\
\text { cha del programa. } \\
\text { Directa con opi- } \\
\text { niones de la comu- } \\
\text { nidad a través de } \\
\text { las redes sociales } \\
\text { de la emisora y en } \\
\text { la intervención en } \\
\text { los pregrabados en } \\
\text { territorio. }\end{array}$} \\
\hline & & $\begin{array}{l}\text { Los niños expresan su vi- } \\
\text { sión de la paz a partir de } \\
\text { vivencias en los entornos } \\
\text { familiar, escolar y de vecin- } \\
\text { dad (arraigo). }\end{array}$ & & \\
\hline "El amor en & & $\begin{array}{l}\text { Énfasis en la importancia } \\
\text { del conflicto y en la exis- } \\
\text { tencia de instituciones que } \\
\text { protejan a los niños. }\end{array}$ & & \\
\hline
\end{tabular}

Fuente: elaboración propia. 
Con respecto a la serie En clave de paz (tabla 3), se encontraron narraciones en las que se buscó un sentido de la paz que trascendiera la academia, pero sin descuidarla. En este sentido, se abordaron los factores sociales que incidían en la paz del entorno, la resolución de conflictos y los modos de consolidar puntos de encuentro. Las rutinas de producción incluyeron el acompañamiento de la fundación Aulas de Paz, una organización de desmovilizados que propició la presencia de invitados. La participación también se dinamizó conforme con los recursos propios como medio digital.

Tabla 3. Contenidos de paz en la serie radial En clave de paz

\begin{tabular}{|c|c|c|c|c|}
\hline \multirow{2}{*}{ Programa/serie } & \multirow{2}{*}{ Actores } & \multicolumn{3}{|c|}{ Contenidos de paz } \\
\hline & & Narrativas & $\begin{array}{l}\text { Rutinas de } \\
\text { producción }\end{array}$ & Participación \\
\hline $\begin{array}{l}\text { Capítulo } 1 . \\
\text { Introductorio }\end{array}$ & $\begin{array}{l}\text { Productor y } \\
\text { coproductor. }\end{array}$ & $\begin{array}{l}\text { Generalidades del progra- } \\
\text { ma, marco histórico del } \\
\text { conflicto. Experiencias } \\
\text { del coproductor en asun- } \\
\text { tos de paz. }\end{array}$ & \multirow{3}{*}{$\begin{array}{l}\text { Programas pre- } \\
\text { grabados con } \\
\text { preproducción } \\
\text { de algunos vox } \\
\text { pop y pregun- } \\
\text { tas de terceros, } \\
\text { intervención } \\
\text { constante de } \\
\text { expertos y acto- } \\
\text { res del conflic- } \\
\text { to. Interacción } \\
\text { con el productor, } \\
\text { quien estable- } \\
\text { ce un libreto y } \\
\text { lo pone a consi- } \\
\text { deración de los } \\
\text { participantes. }\end{array}$} & \multirow{3}{*}{$\begin{array}{l}\text { Indirecta por par- } \\
\text { te de los oyentes } \\
\text { de las comunida- } \\
\text { des invitadas con } \\
\text { la escucha del } \\
\text { programa. } \\
\text { Directa con opi- } \\
\text { niones de la co- } \\
\text { munidad a través } \\
\text { de las redes socia- } \\
\text { les de la emisora y } \\
\text { la intervención en } \\
\text { los pregrabados } \\
\text { en territorio. }\end{array}$} \\
\hline \multirow[b]{2}{*}{$\begin{array}{l}\text { Capítulo } 2 . \\
\text { Apuntes de } \\
\text { historia }\end{array}$} & \multirow{2}{*}{$\begin{array}{l}\text { Productor, } \\
\text { coproduc- } \\
\text { tor, invitado } \\
\text { especial: ex } \\
\text { paramili- } \\
\text { tar Roberto } \\
\text { Duque cono- } \\
\text { cido como } \\
\text { "Ernesto } \\
\text { Báez". }\end{array}$} & $\begin{array}{l}\text { ria y polifónica frente al } \\
\text { conflicto y valoración del } \\
\text { arraigo. }\end{array}$ & & \\
\hline & & $\begin{array}{l}\text { Relatos del conflicto en } \\
\text { clave de justicia y repara- } \\
\text { ción por pare del invitado. }\end{array}$ & & \\
\hline
\end{tabular}

Fuente: elaboración propia.

\section{Discusión}

El alcance de las creaciones radiales se revisa a partir de los cinco principios para los contenidos culturales en la cultura de la paz, propuestos por la Unesco (1999) y el Ministerio de Cultura de la República de Colombia (2016). Además, se enuncian las estrategias construidas con los directores y productores para la sostenibilidad de las realizaciones, y su aporte a la memoria histórica de la localidad. Respecto a lo primero, las generalidades del alcance se precisan en la tabla 4. 
Tabla 4. Alcance de los contenidos de paz en las producciones radiales

\begin{tabular}{|c|c|c|c|}
\hline \multirow{2}{*}{$\begin{array}{c}\text { Programa radial / Princípios } \\
\text { de Unesco y Mincultura }\end{array}$} & $\begin{array}{l}\text { Por las calles de mi ba- } \\
\text { rrio }\end{array}$ & Locutores por la paz & En clave de paz \\
\hline & $\begin{array}{c}\text { San Buenaventura Es- } \\
\text { téreo }\end{array}$ & \multicolumn{2}{|c|}{ Radio B } \\
\hline Diversidad & $\begin{array}{l}\text { La variedad de expre- } \\
\text { siones de grupos en } \\
\text { los barrios. }\end{array}$ & $\begin{array}{l}\text { La interacción con } \\
\text { padres de familias e } \\
\text { hijos. }\end{array}$ & $\begin{array}{l}\text { Reconocimiento de } \\
\text { actores del conflicto. }\end{array}$ \\
\hline Inclusión & $\begin{array}{l}\text { Los barrios conocen } \\
\text { las acciones de los } \\
\text { otros. }\end{array}$ & $\begin{array}{l}\text { Dar prioridad a las } \\
\text { voces infantiles. }\end{array}$ & $\begin{array}{l}\text { Tener en cuenta diver- } \\
\text { sas posturas sobre el } \\
\text { conflicto. }\end{array}$ \\
\hline Participación & $\begin{array}{l}\text { Es efectiva, ya que el } \\
\text { programa es en vivo. } \\
\text { Hay diversidad de ca- } \\
\text { nales de interacción. }\end{array}$ & \multicolumn{2}{|c|}{$\begin{array}{l}\text { Los programas aún son pregrabados. Falta } \\
\text { mayor interacción directa con el público. La } \\
\text { emisora no cuenta con un chat directo, lo cual } \\
\text { es suplido con la página de Facebook, el correo } \\
\text { electrónico y el número telefónico. }\end{array}$} \\
\hline Respeto & $\begin{array}{l}\text { Se comprenden las } \\
\text { diferencias de un ba- } \\
\text { rrio a otro desde la } \\
\text { afirmación de sus ne- } \\
\text { cesidades. }\end{array}$ & $\begin{array}{l}\text { Desde la óptica infan- } \\
\text { til se hacen exigencias } \\
\text { y requerimientos al } \\
\text { Estado y grupos so- } \\
\text { ciales. }\end{array}$ & $\begin{array}{l}\text { Énfasis en la necesi- } \\
\text { dad del diálogo para } \\
\text { la solución de proble- } \\
\text { máticas. }\end{array}$ \\
\hline Diálogo cultural & $\begin{array}{l}\text { Hay visibilización de } \\
\text { las comunidades me- } \\
\text { diante creación de na- } \\
\text { rrativas. }\end{array}$ & $\begin{array}{l}\text { Ejercicio de salida de } \\
\text { la programación en la } \\
\text { cabina hacia realidad } \\
\text { de comunidades ale- } \\
\text { dañas. }\end{array}$ & $\begin{array}{l}\text { Escuchar los disensos } \\
\text { desde la versión de los } \\
\text { mismos actores del } \\
\text { conflicto otorga un } \\
\text { valor reconocible. }\end{array}$ \\
\hline
\end{tabular}

Fuente: elaboración propia.

\section{Diversidad}

Los contenidos de paz construidos en ambas emisoras dan cuenta de las distintas formas de expresión que se encuentran en los barrios del municipio de Bello o en segmentos representativos del mismo. En cuanto a la emisora comunitaria, por su misma condición, guarda una cercanía especial con sus audiencias en la medida en que permite el encuentro de los oyentes desde el sentido de la vecindad. Este hecho incide en las formas de relacionamiento de sus habitantes (Rodriguez y Cadavid, 2007) gracias a la mediación comunicativa que hacen los líderes de los barrios con las personas que representan. En esta medida, se encontraron demandas y valores a resaltar de las comunidades que se pudieron plasmar en los programas. Por su parte, la emisora cultural trata de llegar a diferentes públicos que se ven representados en 
los menores o en los actores de los procesos de paz y la reconciliación. En este caso, la proximidad está dada por el mundo digital y la accesibilidad al mismo.

Las diversas expresiones de paz encontradas en los programas consultados parten y se orientan desde el conflicto, comprendido como disenso de opiniones para contar con un panorama amplio sobre las problemáticas significativas tanto para los productores como para las audiencias. De ahí que se reconfigure como sentido de cooperación más que de competición (Villanueva y Ortiz, 2013), lo que representa un beneficio común para todas las personas implicadas. Desde este valor del disenso, las tres producciones abordan un concepto de paz "positiva" según Galtung (2014). Esta consiste en una transformación creativa y no violenta del conflicto que hace frente a la paz negativa, la cual se relaciona con la ausencia o reducción de todo tipo de violencia. Esto pudo notarse en la publicidad de problemáticas de los barrios, las cuales son vistas como oportunidades de trabajo comunitario con el apoyo estatal. En las propuestas de los niños, se evidencia la paz positiva cuando se intenta crear un ambiente más amigable en los hogares y los entornos cercanos con problemáticas sociales generalizadas. Incluso, esta se manifiesta en la postura afirmativa de la paz en la voz de sus protagonistas, como lo pudieron experimentar las directivas de la emisora cultural.

\section{Inclusión}

La valoración positiva de las diferencias aparece en los tres programas a partir de las perspectivas del tipo de actores en el conflicto y su respectivo grupo etario. Por las calles de mi barrio y En clave de paz son producciones de adultos, con quienes se realizan procesos de construcción de memoria histórica, se establecen conceptos propios de paz y se proponen alternativas para la mejora de las condiciones estructurales del entorno bellanita. En este sentido, estos programas se acercan a la destacada experiencia Sintonizate con la paz de la Fundación Arco Iris (Señal Colombia, 2016). Así, desde la forma de construir los conceptos de paz, revelan también formas particulares de producción: Por las calles de mi barrio no se reduce a una sola localidad o JAC, sino que da la posibilidad de expresión a los que se deseen vincular. En el caso de En clave de paz, el esfuerzo de sus productores consiste en integrar los ejes conceptual y vivencial del conflicto para contar con una mirada interdisciplinar sobre el tema mediante la contextualización propia de la realidad social del municipio de Bello y el apoyo de la Fundación Aulas de Paz.

La idea del trabajo sobre la paz con población infantil se ubica en la línea de los antecedentes mencionados (Señal Colombia, 2016). La diferencia es que, en todos ellos, se tratan temas de participación y de la producción de la radio como tal, pero no de la cultura de la paz, que es en donde se inscribe la producción de Radio B. El 
ejercicio de formación directa de Radio B sobre el tema de la paz trasciende los micrófonos de la emisora y lo acerca a otras iniciativas en el país. Si bien no se usa el medio radial, se enfatiza en la construcción de la paz desde la postura de los niños. En este sentido, se configura la propuesta del Centro Internacional de Educación y Desarrollo (Cinde) denominada Niños, niñas y jóvenes constructores de paz. Allí la paz es el producto de la construcción en la que los sujetos, en este caso los niños, desempeñan un papel activo con el fomento de la capacidad argumentativa y de toma de decisiones en pro de un mayor bienestar social (Cinde, 2017). Asimismo, se encuentra el programa Aulas en paz (Chaux et al., 2017), liderado por la Universidad de los Andes. Este programa es de tipo "multicomponente" en tanto trabaja en la prevención de la agresión y la promoción de la sana convivencia mediante el trabajo con docentes, estudiantes y familias. Estas formas de inclusión hablan por sí mismas de lo que los contenidos de la cultura de paz deben promover: dejar atrás los extremos ideológicos y los enojos, donde la radio debe asumir el papel social que le compete (López y Arriola, 2015).

Las oportunidades que brindan los medios radiales a los habitantes del municipio para hacer efectivo su derecho constitucional a la participación, están en consonancia con la trayectoria de la localidad en la promoción y trabajo de los movimientos sociales y la cultura (Secretaría de Educación y Cultura de Bello, 2015). Los líderes formados en las diferentes etapas de desarrollo cultural en Bello se convierten en promotores de procesos de memoria histórica y gestores de nuevos relatos para sus barrios. Tienen el objetivo de legar a las generaciones venideras su preocupación por el fomento del capital social en cada sector, lo cual se puede revisar en los tres ámbitos propuestos por el Ministerio de Cultura para la vinculación activa de los habitantes en los proyectos radiales. El primero es el fomento de habilidades para la vida que genera la cultura de la paz. Los líderes comunitarios asumen su papel de voceros y operan, en compañía de sus vecinos y coadyuvando en el newsmaking de la paz, como gatekeepers o seleccionadores de información relevante de sus sectores. Por el otro lado, los niños aprenden producción radial y dan pasos en la planificación de temas sobre convivencia. Los actores del conflicto, por su parte, intervienen de forma propositiva al convertirse en cocreadores de los programas radiales. De esta manera, se ejercitan en acciones que afianzan cada vez más su sentido humano desde sus capacidades, según lo busca el Objetivo de Desarrollo Sostenible número cuatro (Naciones Unidas, 2015), el cual propicia desarrollo humano y sostenible. El segundo ámbito tiene que ver con la construcción de ciudadanía; la vinculación activa de los coproductores los ubica en el tipo de ciudadano comprometido con la justicia, más allá de ser solo personalmente responsable o participativo (Westheimer y Kahne, 2004). Por su parte, el tercer ámbito sugerido son las condiciones de circulación y consumo de las creaciones radiales en condiciones de equidad. Si bien este aspecto es favorecido por ambas emisoras al garantizar la igualdad a participantes y oyentes en la creación y producción, se da 
una afectación a las mismas por el hecho de ser todavía pregrabadas, ya que faltan mayores oportunidades de interactividad que posibiliten a los oyentes ser escuchado in situ. Esta falencia tiene que ver con la estructura propia de los medios, los recursos con que cuentan y las dificultades de agendas de los involucrados.

\section{Respeto}

La aceptación de las diferencias entre las personas se ve reflejada en los modos en que los medios radiales se acercan al concepto de violencia; este no es unívoco y ha sido favorable para tratar de forma amplia la cuestión de la paz. En términos de Pecaut (2006), ha permitido generar un amplio espectro de ámbitos de análisis superior que el que posibilitaba el concepto de guerra. Precisamente, el direccionamiento que se ha dado a los programas aborda el tema de las diferentes violencias que se dan en las múltiples formas de interrelación entre las personas, según sus espacios compartidos y en donde la radio opera como una gramática alternativa que las dota de significado y les otorga reconocimiento y respeto (Acosta, 2019). Esto pudo apreciarse en las expresiones de los niños acerca de las necesidades para la sana convivencia en sus entornos próximos, los testimonios de excombatientes en clave de justicia y reparación y los reclamos de los líderes comunales al Estado en aras de garantías de no repetición y sostenibilidad de la paz en sus barrios. Esta forma de tratar el concepto de violencia la acerca al tipo de paz cultural en términos de Galtung (2015). Este tipo de paz se relaciona con actitudes que legitiman la primera, entre las que destaca la necesidad de transformar el lenguaje y los significantes empleados con respecto al conflicto y sus implicaciones.

\section{Diálogo cultural}

Las experiencias radiales para la paz de ambos medios han creado nuevas narrativas en las localidades del municipio. En este sentido, construyen imaginarios diferentes a los que solían tener sobre Bello a causa de los medios de comunicación masiva. Estos nuevos relatos parten de la necesidad de edificar la paz desde acciones sutiles, desde la cotidianidad del hogar, la escuela, las relaciones entre vecinos y la reconciliación con los victimarios. Para ello, se rememora el conflicto desde una postura reconciliadora en pro de la salud mental del colectivo social. Ruiz y Sánchez (2012), citando a Galtung (1998), plantean que existen dos modelos o paradigmas periodísticos a la hora de abordar los conflictos: el periodismo de guerra dominante y el periodismo de paz alternativo. El periodismo alternativo o de paz cuenta con unas características propias, como la orientación hacia la paz, la verdad civil y la solución de conflictos. Precisamente, sobre este tipo de periodismo se han enfocado las producciones abordadas para extender su aún inicial campo de acción. De esta manera, se genera identidad entre los habitantes y, sobre todo, en relación con la apropiación del terri- 
torio, en consonancia con las investigaciones similares consultadas (Calderón, 2015; Espinar y Hernández, 2012; Gouse et al., 2019). El arraigo es uno de los logros más significativos de las producciones radiales porque, a la vez, es el eje estructural para la continuidad de la radio en la naciente cultura de la paz en el municipio.

\section{Estrategias de sostenibilidad de las producciones para contribuir a la memoria histórica del municipio de Bello}

En la línea de la construcción de paz, los directores y productores de ambas emisoras establecieron propuestas para generar sinergias con la academia en pro de la sostenibilidad de las producciones radiales de paz y su aporte a la memoria del conflicto desde la localidad. Mediante un taller de diseño participativo, se enfatizaron aspectos como los encontrados en las experiencias de otras radios indagadas: la acción mancomunada con los comités editoriales y juntas de programación para el diseño de agendas diversas y compartidas (González y Rodríguez, 2006; Cadavid y Moreno, 2009). De esta manera, se dio apertura a todos los sectores sociales para evitar silenciar testimonios que pudieran desfavorecer la comunicación de la paz. Asimismo, hay una amplia participación de la comunidad, como ha sucedido con las emisoras comunitarias (Zúñiga y Grattan 2017), pero que tiene también acogida en un medio cultural y digital como Radio B. Los participantes tomaron decisiones que convinieran a las dos generaciones implicadas en el momento actual de posacuerdo que viven las emisoras: de un lado, los adultos vinculados como reporteros y ciudadanos activos a partir de sus roles de líderes barriales, generadores de opinión de colectivos sociales, comunicadores empíricos, aficionados asociados con programas específicos o solo oyentes. Para ello se consideró preservar y potenciar su vínculo mediante formación y trabajo conjunto con los investigadores, con el ánimo de resignificar las voces de las vivencias pasadas de la violencia que permiten la reconciliación actual (Gaborit, 2006). De otro lado, el público adolescente y juvenil, característica común a las dos emisoras, fue considerado como nicho propicio para encaminar rutas de trabajo a mediano plazo. En este sentido, la propuesta construida se encaminó a generar dos dinámicas importantes para la emisora y la comunidad: espacios de participación y construcción por parte de ellos, desde una mirada inclusiva y plural de la emisora en su programación de contenidos. Asimismo, se perfilaron como dinámicas de participación y ejercicio ciudadano del derecho a la comunicación; todo ello con el acompañamiento de la universidad implicada como parte del proceso investigativo. En el caso de San Buenaventura Estéreo, dada su presencia física en una institución educativa, se contempló el vínculo de programación juvenil realizada por los estudiantes, con temas y abordajes en los que ellos relataran sus concepciones de paz. Además, se propició la escucha atenta a los testimonios de sus pares, familiares, amigos y vecinos con respecto a que las narrativas positivas permitieran afianzar la identidad institucional 
en el contexto del posacuerdo como entorno generador de convivencia y creador de memoria histórica en el municipio. Esta situación fue similar a la de Radio B y su relación intrínseca con la Escuela de Música de Bello. Como este espacio cultural surgió como una alternativa juvenil a la situación de violencia estructural en la localidad, se precisó afianzar los espacios radiales ya existentes. Así, se contó con participación de los músicos en formación para crear otros medios alternativos de mayor apropiación que permitieran contar con nuevos discursos en los que la experiencia artística recreara el conflicto de Bello desde las dinámicas generadas por el quehacer musical y las implicaciones en el entorno de los participantes. Estos espacios habrían de convertirse en semilleros formativos para aprovechar el terreno ganado en Radio B con los niños, y brindarles formación radial a los menores para lograr producciones radiales con un desempeño todavía más amplio que el logrado hasta ahora.

Por último, fueron resaltadas las dificultades operativas con las que debían sortear la compatibilidad de sus agendas con las disposiciones y regulaciones del Estado (Vega, Tapias y Pérez, 2019), y asuntos de infraestructura que implicaban la no accesibilidad a los medios radiales. Entre los primeros se destacó el aprovechamiento de las TIC y esfuerzos para lograr la producción de contenidos en los propios territorios y en directo. De esta manera, se favoreció la posibilidad de contar con las grabaciones de los programas de forma digital, abierta y desde formas de almacenamiento de acceso permanente. Este aspecto fue resaltado como una ventaja importante para garantizar el derecho a la información y a la comunicación de mensajes positivos en favor de la no repetición y la conciencia colectiva de preservar los valores pacíficos y de convivencia construidos. Frente a la infraestructura, se habló de la poca accesibilidad a la señal en muchos sectores del municipio para escuchar la emisora comunitaria, además de otros problemas de conectividad y cultura digital en el caso de la emisora cultural. Como medidas a corto plazo, se mencionó el proceso de reubicación de una de las antenas repetidoras de señal para ampliar la cobertura y el aumento en la conectividad para el municipio de la mano de programas de telecomunicaciones en sectores estratégicos y comunes de la localidad. Mediante un documento quedaron plasmadas estas iniciativas y se estableció un cronograma de trabajo compartido para su aplicación y medición de impactos.

\section{Conclusiones}

La caracterización de los procesos de construcción de contenidos de paz en los dos medios radiales del municipio de Bello cuenta con programación propia. Asimismo, siguen las directrices sugeridas por parte de organismos como la ONU y el Ministerio de Cultura de Colombia en pro de la creación de relatos que coadyuvan a la memoria histórica de esta localidad. De este modo, la emisora comunitaria San Buenaventura Estéreo y la emisora cultural y digital Radio B, siguen dichas orientaciones y llevan 
a término el propósito de los acuerdos de paz en tanto promueven la participación ciudadana y fomentan la cultura de paz. Con esfuerzos notables por parte de sus directores en la promoción de dicha cultura, han abierto sus micrófonos para que, conforme con la orientación del medio, se enfatice en el compromiso ciudadano de construir nuevas narrativas de paz que conllevan a la apropiación significativa de un territorio que ha sido estigmatizado como violento por los medios de comunicación masiva y por su situación social bajo matices recurrentes. Frente a ello, los nuevos relatos propuestos desde las creaciones radiales, coadyuvan a la construcción de la memoria histórica de los bellanitas. En estos relatos se ponen en juego las capacidades de los ciudadanos para contar, con una mirada particular, la vivencia de sus propias violencias frente a la guerra generalizada como situación común en el municipio. Las narrativas emergentes, inscritas como nuevas gramáticas que validan las voces de quienes no habían hablado, no solo les hacen justicia al ser comunicadas, sino que trascienden lo retórico. De este modo, los ciudadanos son proactivos al generar alternativas que hacen sostenible la paz que se logra en sus entornos más cercanos y que buscan devolver el statuo quo pacífico que tenía su territorio mediante los aprendizajes que les deja el conflicto. Es en este contexto donde las voces de los victimarios son recontextualizadas en el sentir de las víctimas.

La producción de contenidos de paz con los públicos participantes fue identificada en la labor de los medios y sus rutinas. Se encontró como constante en las tres producciones abordadas la preocupación por el arraigo y el fomento de las capacidades endógenas de las comunidades o de sus actores para hacer frente a las distintas violencias con las que cuenta el municipio. A la vez, fue notoria la disposición a contribuir en la construcción de guiones radiales y aprender del quehacer de este medio; todo lo cual, constituye aprendizajes para la vida que proveen de desarrollo humano y sostenible tanto a ellos como a sus audiencias. Las rutinas incluyeron el uso de técnicas de recolección de información con la comunidad, opiniones de expertos y articulación con los productores directos de las emisoras. No obstante, las dificultades estructurales no han permitido la emisión en vivo. Esta situación resta fuerza a la participación, la cual se hace de forma directa con la escucha de las producciones o como fuentes del programa; y de forma indirecta, mediante los canales de retroalimentación con que se cuenta, preferiblemente de manera paralela, como las redes sociales.

En consonancia con la investigación constructiva de la paz, se analizaron los aspectos encontrados en los contenidos de las dos emisoras, como teorías factibles, con el deber ser emanado por las indicaciones de instituciones de carácter nacional y foráneo, citadas en esta materia. Mediante la revisión de cinco aspectos claves, se evidenció que las creaciones radiales cumplían de modo satisfactorio con cuatro de ellos: diversidad, donde la paz es vista como transformación creativa y no violenta del conflicto para dar la voz a todos; inclusión, mediante la cual las oportunidades de 
participación retoman la trayectoria de liderazgo propia del municipio; respeto, como escucha atenta desde la gramática radial para abordar diferentes violencias conforme a las interrelaciones de los bellanitas; y diálogo cultural, gestionado a partir del valor del arraigo y la remembranza del conflicto en pro de la salud mental social. No sucedía lo mismo con el aspecto de la participación, ya que las condiciones estructurales de los medios, además del cruce de agendas con los participantes, no permitían contar con grabaciones en vivo que favorecieran niveles altos de interactividad con los oyentes.

Finalmente, los directores y productores radiales propusieron acciones de trabajo conjunto con la academia para garantizar la sostenibilidad de sus elaboraciones y su contribución a la memoria histórica del municipio de Bello. Además de la atención a asuntos técnicos y de infraestructura para garantizar el acceso a los medios radiales, las estrategias fueron orientadas a los dos públicos presentes en el momento actual del posacuerdo: de un lado, los mayores como actores implicados en la producción como reporteros ciudadanos o creadores de contenidos; con ellos se establecieron planes de acción en formación y acompañamiento por parte de los investigadores asociados. De otro lado, los adolescentes y jóvenes se asociaron a ambas emisoras por su mismo carácter, de modo que se convirtieran en realizadores de contenidos de paz, a partir de sus propias vivencias, con las personas de su entorno cercano desde la perspectiva de actores escolares o músicos en formación, respectivamente. Su labor habría de generar semilleros de producción radial para niños, con lo que se aprovecharían los beneficios obtenidos con esta audiencia por parte de la emisora cultural.

Queda por mirar el impacto de estas estrategias y revisar su aporte a la línea investigativa de construcción de la paz como referentes que podrán contrastarse con otras producciones radiales en esta materia; todo ello en el contexto del posacuerdo, donde la radio trata de iluminar su horizonte de comprensión desde formas de escucha significativas.

\section{Referencias}

Acosta, M. (2019). Grammars of listening philosophical approaches to the construction of historical memory. Gramáticas de la escucha, 68(5), 59-79.

Ali, M. (2012). Comunicar la paz. Estudios sobre comunicación y medios para las comunidades. Editorial Académica Española.

Alto Comisionado para la Paz. (2016). Acuerdo Final para la Terminación del Conflicto y la Construcción de una Paz Estable y Duradera. https://www.cancilleria.gov.co/sites/default/files/Fotos2016/12.1 1_1.2016nuevoacuerdofinal.pdf

Belalcázar, J., Botero, Y. y Ospina, M. (2019). Emisora comunitaria Voces Montañeras Tenjo Colombia: entre la desterritorialización del conflicto armado y la re-territorializacion de la paz. Algarrobo-MEL, 7, 1-23. 
Cadavid, A. y Moreno, O. (2009). Evaluación cualitativa de radio audiencias por la paz en el Magdalena Medio colombiano. Signo y Pensamiento, 28(54), 276-299.

Calderón, J. (2015). La cobertura informativa de los medios de comunicación frente al conflicto armado y el proceso de paz en Colombia: responsabilidad frente a la divulgación de la noticia. Ciudad Paz-Ando, 7(2), 92-112.

Caridad, M., Peña, J., Blanco, E., Cruz, C., Franco, B., y Echeverría, R. (2017). Resolución de conflictos para el fomento de la cultura de paz: Importancia de los medios de comunicación alternos en Colombia. Revista Lasallista de Investigación, 14(1), 56-65.

Centro Internacional de Educación y Desarrollo Humano, Cinde. (2017). Niños, Niñas y Jóvenes Constructores-as de Paz, una propuesta para fortalecer subjetividades políticas y generar procesos de construcción de Paz. Revista Latinoamericana de Ciencias Sociales, Niñez y Juventud, 15(2), 1390-1396.

Cerdas-Agüero, E. (2015). Desafíos de la educación para la paz hacia la construcción de una cultura de paz. Revista Electrónica Educare, 19(2), 135-154.

Chaux, E., Barrera, M., Molano, A., Velásquez, A. M., Castellanos, M., Chaparro, M. P. y Bustamante, A. (2017). Classrooms in peace within violent contexts: Field evaluation of aulas en paz in colombia. Prevention Science, 18(7), 828-838. https://doi.org/10.1007/s1 1121 017-0754-8

Escuela de Cultura de Paz. (2011). Educar para una cultura de paz. Cuadernos de construcción para la paz, 1 26.

Espinar, E. y Hernández, M. I. (2012). El periodismo de paz como paradigma de comunicación para el cambio social: características, dimensiones y obstáculos. Cuadernos de Información y Comunicación, 17,175-189.

Espinoza, A. R. (2017). La comunicación para la paz como herramienta de transformación social. Vox Juris, 33(1), 143-157.

Espitia, N. (2018). Emisoras comunitarias para la paz, Colombia 2018. Expresiones, Revista Estudiantil de Investigación, 6(11), 36-44.

Gaborit, M. (2006). Memoria Histórica: Relato desde las víctimas. Revista Pensamiento Psicológico, 2(6), 7-20.

Galtung, J. (2008). Encyclopedia of violence, peace, and conflict. Elsevier.

Galtung, J. (2014). Peace, conflict, and violence. En H. Hintjens y D. Zarkov (eds.). Conflict, peace, security and development: Theories and methodologies (pp. 25-38). Routledge.

Galtung, J. (2015). Peace. International encyclopedia of the social \& behavioral sciences: Second edition (pp. 618 623). https://www.sciencedirect.com/science/article/pii/B9780080970868960197?via\%3Dihub

González, A. y Rodríguez, C. (2006). Alas para tu voz. Ejercicios de Ciudadanía desde una emisora comunitaria. Belén de los Andaquies. Centro de Competencia en Comunicación para América Latina.

Gouse, V., Valentin-Llopis, M., Perry, S. y Nyamwange, B. (2019). An investigation of the conceptualization of peace and war in peace journalism studies of media coverage of national 
and international conflicts. Media, War and Conflict, 12(4), 435-449. https://www.semanticscholar. org/paper/An-investigation-of-the-conceptualization-of-peace-Gouse-Valentin-Llopis/55740 259bac9ae2e8d072b64371 1b563a97ac7bb

Kalman, J. y Rendón, V. (2016). The use of spreadsheet to analyse qualitative data. [Uso de la hoja de cálculo para analizar datos cualitativos] Magis, 9(18), 29-48. https://doi.org/10.11144/ Javeriana.m9-18.uhca

López, J. y Arriola, T. (2015). Módulo de producción radial. Claves para la producción de contenidos de paz. https:// www.mincultura.gov.co/areas/comunicaciones/fortalecimientodelaradiocomunitariaypublica/ diplomadose\%C3\%Blalesdepaz/Documents/MODULO\%20PRODUCCION\%20RADIAL.pdf

Ministerio de Cultura de la República de Colombia. (2016). Módulo Comunicación y cultura de paz. Comunicación en tiempos de no guerra: fomento a la cultura de la paz. https://www. mincultura.gov.co/areas/comunicaciones/fortalecimientodelaradiocomunitariaypublica/ diplomadose\%C3\%Blalesdepaz/Documents/M\%C3\%93DULO\%20COMUNICACI\%C3\%93N\%20 Y\%20CULTURA\%20DE\%20PAZ.pdf

Moreno, M. (2019). Comunicar para reconstruir: papel de la emisora comunitaria Simití Original Estéreo en la reconstrucción de la memoria y la promoción de una cultura de paz en el municipio de Simití, Bolívar, luego de la firma del Acuerdo de Paz del 2016 [tesis de maestría, Pontificia Universidad Javeriana] Repositorio Universidad Javeriana.

Muñoz, F. y Molina B. (2010). Una Cultura de Paz compleja y conflictiva. La búsqueda de equilibrios dinámicos. Revista de Paz y Conflictos, (3), 44-61. https://www.redalyc.org/articulo. oa?id=205016387004

Naciones Unidas. (2015, 25 de septiembre). Transformar nuestro mundo: la agenda 2030 para el Desarrollo Sostenible. Resolución aprobada por la Asamblea General.

Pecaut, D. (1997). Presente, pasado y futuro de la violencia en Colombia. Desarrollo Económico, 36(144), 891-929.

Pecaut, D. (2006). Crónica de cuatro décadas de política colombiana. Editorial Norma.

Reyes, M. (2017). La Radio Comunitaria como herramienta comunicativa en La construcción de paz en el campesinado de la vereda San Jorge del municipio de Soacha Itesis de pregrado, Corporación Universitaria Minuto de Dios]. Repositorio Uniminuto.

Rodriguez, C. y Cadavid, A. (2007). From violence to discourse: Conflict and citizens' radio stations in Colombia. En H. Anheier y Y. Raj (eds.). Conflicts and tensions (pp. 313-327). Sage.

Ruiz, E. y Sánchez, M. (2012). El periodismo de paz como paradigma de comunicación para el cambio social: características, dimensiones y obstáculos. Cuadernos de Información y Comunicación, 17, 175-189.

Secretaría de Educación y Cultura de Bello, (2015). Plan Decenal de Cultura 2015 - 2025. Municipio de Bello. Subsecretaría de Cultura de Bello "Para tejer sentidos de ciudadanía". https://issuu.com/ labitacorabello/docs/plan_municipal_de_cultura_bello_201

Señal Colombia. (2016). Programas de radio donde los niños son protagonistas. https://www.misenal.tv/ noticias/_programas-de-radio-donde-los-ninos-son-protagonistas 
Taylor, S. y Bodgan, R. (1986). Introducción a los métodos cualitativos de investigación. Paidós Ibérica.

Unesco. (1999). Declaración sobre una cultura de paz. http://www.fund-culturadepaz.org/doc/ Declaracion-CulturadePaz-FacilLectura.pdf

Useda, E. y Carillo, J. (2018). El impacto sociocultural de las nuevas tecnologías en los programas radiales en comunidades indígenas: las palabras de resistencia que tienen frecuencia en territorios de paz. Adaya Press.

Vega J. y Bayuelo, S. (2008). Ganándole terreno al miedo: cine y comunicación en Montes de María. En C. Rodríguez (ed.), Lo que le vamos quitando a la guerra. Medios ciudadanos en contextos de conflicto armado en Colombia. Centro de Competencias en Comunicación, Fundación Friedrich Ebe.

Vega, J., Tapias, C. y Pérez, C. (2019). Radio comunitaria y construcción de paz en Colombia. Comunicación, interacción y planeación participativa para el posconflicto. Revista Latina de Comunicación Social, 74, 1391-1410.

Vega, J. y Rodríguez, C. (2009). Citizens' media as political subjects: The case of community radio in Colombia. En D. Kishan (ed.). Internationalizing media studies (pp. 228-245). Routledge Taylor y Francis Group.

Villanueva I. y Ortiz, C. M. (2013). Desarrollo de la cultura de la paz y la convivencia en el ámbito municipal: La mediación comunitaria. Política y Sociedad, 50(1), 179-194.

Westheimer, J. y Kahne, J. (2004). What kind of citizen? the politics of educating for democracy. American Educational Research Journal, 41(2), 237-269. https://doi.org/10.3102/00028312041002237

Zúñiga, D. y Grattan, S. (2017, 14 de septiembre). Papel de las radios comunitarias en el proceso de consolidación de la paz en Colombia. Humanidades digitales, diálogo de saberes y prácticas colaborativas en red [ponencia]. Cátedra Unesco de Comunicación, Bogotá, Colombia. https://www.javeriana. edu.co/unesco/humanidadesDigitales/ponencias/IV_24.html 